\title{
A Proximal Alternating Direction Method of Multipliers with a Substitution Procedure
}

\author{
Miantao Chao $\mathbb{D D}^{1}{ }^{1}$ Yongxin Zhao, ${ }^{1}$ and Dongying Liang $\mathbb{D}^{2}$ \\ ${ }^{1}$ Department of Mathematics, Guangxi University, Nanning 530004, China \\ ${ }^{2}$ Guangxi Vocational and Technical College of Communications, Nanning 530023, China
}

Correspondence should be addressed to Dongying Liang; liangdy_go@126.com

Received 27 September 2019; Accepted 27 March 2020; Published 27 April 2020

Academic Editor: Jerzy Baranowski

Copyright ( $\odot 2020$ Miantao Chao et al. This is an open access article distributed under the Creative Commons Attribution License, which permits unrestricted use, distribution, and reproduction in any medium, provided the original work is properly cited.

\begin{abstract}
In this paper, we considers the separable convex programming problem with linear constraints. Its objective function is the sum of $m$ individual blocks with nonoverlapping variables and each block consists of two functions: one is smooth convex and the other one is convex. For the general case $m \geq 3$, we present a gradient-based alternating direction method of multipliers with a substitution. For the proposed algorithm, we prove its convergence via the analytic framework of contractive-type methods and derive a worst-case $O(1 / t)$ convergence rate in nonergodic sense. Finally, some preliminary numerical results are reported to support the efficiency of the proposed algorithm.
\end{abstract}

\section{Introduction}

In this paper, we consider the following convex minimization model with linear constraints and separable objective function:

$$
\min \left\{\sum_{i=1}^{m}\left[f_{i}\left(x_{i}\right)+g_{i}\left(x_{i}\right)\right] \mid \sum_{i=1}^{m} A_{i} x_{i}=b, x_{i} \in X_{i}, i=1, \ldots, m\right\},
$$

where $f_{i}: \mathscr{R}^{n_{i}} \longrightarrow \mathscr{R} \cup\{+\infty\}(i=1, \ldots, m)$ are closed proper convex functions and $g_{i}: \mathscr{R}^{n_{i}} \longrightarrow \mathscr{R}(i=1, \ldots, m)$ are smooth convex functions, $\mathscr{X}_{i} \subseteq \mathscr{R}^{n_{i}}(i=1, \ldots, m)$ are closed convex sets, $A_{i} \in \mathscr{R}^{l \times n_{i}}(i=1, \ldots, m)$ are given matrices, and $b \in \mathscr{R}^{l}$ is a given vector. Furthermore, we assume that each $g_{i}$ has Lipschitz-continuous gradient, i.e., there exists $L_{i}>0$ such that

$$
\left\|\nabla g_{i}(x)-\nabla g_{i}(y)\right\| \leq L_{i}\|x-y\| \text {, for all } x, y \in \mathscr{X}_{i} .
$$

Throughout the paper, the solution set of (1) is assumed to be nonempty.

A fundamental method for solving (1) in the case of $m=2$ is the alternating direction method of multipliers (ADMM), which was presented originally in $[1,2]$. We refer to $[3,4]$ for some review papers on ADMM. There are many problems of form (1) with $m \geq 3$ in contemporary applications, such as the robust principal component analysis model [5], the total variation-based image restoration problem [6], the superresolution image reconstruction problem $[7,8]$, the multistage stochastic programming problem [9], the deblurring Poissonian images problem [10], the latent variable Gaussian graphical model selection [11], the quadratic discriminant analysis model [12], and the electrical engineering [13, 14]. Then, our discussion focuses on (1) in the case of $m \geq 3$.

A natural idea for solving (1) is to extend the ADMM from the special case $m=2$ to the general case $m \geq 3$. This straightforward extension can be written as follows: 


$$
\left\{\begin{array}{l}
x_{i}^{k+1} \in \underset{x_{i} \in X_{i}}{\arg \min }\left\{f_{i}\left(x_{i}\right)+g_{i}\left(x_{i}\right)+\frac{1}{2} \| \sum_{j=1}^{i-1} A_{j} x_{j}^{k+1}+A_{i} x_{i}\right. \\
\left.+\sum_{j=i+1}^{m} A_{j} x_{j}^{k}-b-\mu \lambda^{k} \|_{H}^{2}\right\}, \quad i=1,2, \ldots, m, \\
\lambda^{k+1}=\lambda^{k}-H\left(\sum_{j=1}^{m} A_{j} x_{j}^{k+1}-b\right) .
\end{array}\right.
$$

The convergence of (3) is proved in some special cases (see [15-17]). Unfortunately, without further conditions, the direct extension of ADMM (3) for the general case $m \geq 3$ may fail to converge (see [18]). In $[19,20]$, the authors present two convergent semiproximal ADMM for two types of 3-block problems. Recently, He et al. [21] showed that if a new iterate is generated by correcting the output of (3) with a substitution procedure, then the sequence of iterates converges to a solution of (1). Since then, several variants of the ADMM were proposed for solving (1) (see [21-26]).

In (3), all the $x_{i}$-related subproblems are in the form of

$$
\min \left\{f_{i}\left(x_{i}\right)+g_{i}\left(x_{i}\right)+\frac{1}{2}\left\|A_{i} x_{i}-a_{i}\right\|_{H}^{2} \mid x_{i} \in \mathscr{X}_{i}\right\} \text {, }
$$

with a certain known $a_{i} \in \mathscr{R}^{l}$ and a symmetric positive definite matrix $H$. When $A_{i}$ is not the identity matrix, problem (4) becomes complicated. A popular technique is to linearize the quadratic term of (4) (see $[27,28]$ ), that is, one can solve the following problem instead of (4):

$$
\min \left\{f_{i}\left(x_{i}\right)+g_{i}\left(x_{i}\right)+\frac{1}{2}\left\|\tau_{i} x_{i}-c_{i}\right\|^{2} \mid x_{i} \in \mathscr{X}_{i}\right\}
$$

with a certain known $c_{i} \in \mathscr{R}^{l}$. In general, one can solve the following problem instead of (4):

$$
\min \left\{f_{i}\left(x_{i}\right)+g_{i}\left(x_{i}\right)+\frac{1}{2}\left\|A_{i} x_{i}-a_{i}\right\|_{H}^{2}+\frac{1}{2}\left\|x_{i}-x_{i}^{k}\right\|_{G_{i}}^{2} \mid x_{i} \in x_{i}\right\},
$$

where $x_{i}^{k}$ is the current iteration. If $G_{i}=\tau_{i} I_{i}-A_{i}^{T} H A_{i}>0$, then (6) becomes the form of (5). (6):

Since $g_{i}$ is smooth, the following problem is easier than

$$
\begin{aligned}
& \min \left\{f_{i}\left(x_{i}\right)+\left\langle\nabla g_{i}\left(x_{i}^{k}\right), x_{i}-x_{i}^{k}\right\rangle+\frac{1}{2}\left\|A_{i} x_{i}-a_{i}\right\|_{H}^{2}+\frac{1}{2}\right. \\
& \left.\cdot\left\|x_{i}-x_{i}^{k}\right\|_{G_{i}}^{2} \mid x_{i} \in x_{i}\right\} .
\end{aligned}
$$

Now, we can give the gradient-based ADMM (GADMM) iterative scheme as follows:

$$
\left\{\begin{array}{l}
\bar{x}_{i}^{k}=\underset{x_{i} \in X_{i}}{\arg \min }\left\{f_{i}\left(x_{i}\right)+\left\langle\nabla g_{i}\left(x_{i}^{k}\right), x_{i}-x_{i}^{k}\right\rangle+\frac{1}{2} \| \sum_{j=1}^{i-1} A_{j} \bar{x}_{j}^{k}+A_{i} x_{i}\right. \\
\left.+\sum_{j=i+1}^{m} A_{j} x_{j}^{k}-b-H^{-1} \lambda^{k}\left\|_{H}^{2}+\frac{1}{2}\right\| x_{i}-x_{i}^{k} \|_{G_{i}}^{2}\right\}, \quad i=1, \ldots, m, \\
\bar{\lambda}^{k}=\lambda^{k}-H\left(\sum_{j=1}^{m} A_{j} \bar{x}_{j}^{k}-b\right) .
\end{array}\right.
$$

In this paper, imal ADMM with a substitution based on (8). In Section 2, we provide some preliminaries for further analysis. Then, we present the gradient-based alternating direction method of multipliers with a substitution (G-ADMM-S) for solving (1) and its convergence is shown in Section 3. In Section 4, we estimate the worstcase iteration complexity for the proposed algorithm in nonergodic sense. In Section 5, some preliminary numerical results are reported to support the efficiency of the proposed algorithm. Finally, some conclusions are given in Section 6.

\section{Preliminaries}

In this section, we provide some preliminaries. Let $\langle x, y\rangle=$ $x^{T} y$ and $\|x\|=\sqrt{\langle x, x\rangle} . G>0(\geqslant 0)$ denotes that $G$ is a positive definite (semidefinite) matrix. For any positive definite matrix $G$, we denote $\|\cdot\|_{G}$ as the $G$-norm. If $G$ is the product of a positive parameter $\beta$ and the identity matrix $I$, i.e., $G=\beta I$, we use a simpler notation: $\|\cdot\|_{G}=\|\cdot\|_{\beta}$. Let $f: \mathscr{R}^{n} \longrightarrow(-\infty,+\infty]$. The domain of $f$ denoted by $\operatorname{dom} f:=\left\{x \in \mathscr{R}^{n} \mid f(x)<+\infty\right\}$. We say that $f$ is convex if 
$f(t x+(1-t) y) \leq t f(x)+(1-t) f(y), \quad \forall x, y \in \mathscr{R}^{n}, \forall t \in[0,1]$.

For convex function $f$, the subdifferential of $f$ is the setvalued operator defined by

$$
\partial f(x):=\left\{\xi \in \mathscr{R}^{n} \mid f(y) \geq f(x)+\langle y-x, \xi\rangle, \quad \forall y \in \operatorname{dom} f\right\} .
$$

2.1. Variational Characterizations of (1). Let $\Theta_{i}\left(x_{i}\right)=$ $f_{i}\left(x_{i}\right)+g_{i}\left(x_{i}\right), \quad i=1,2, \ldots, m, \quad$ and $\mathscr{W}:=\mathscr{X}_{1} \times$ $\mathscr{X}_{2} \times \cdots \times \mathscr{X}_{m} \times \mathscr{R}^{l}$. Since all $\Theta_{i}\left(x_{i}\right)$ are convex functions, by invoking the first-order necessary and sufficient condition for convex programming, one can easily find out that problem (1) is characterized by the following variational inequality: we obtain $w^{*}=\left(x_{1}^{*}, x_{2}^{*}, \ldots, x_{m}^{*}, \lambda^{*}\right) \in \mathscr{W}$ and $\xi_{i}^{*} \in \partial f_{i}\left(x_{i}^{*}\right)(i=1,2, \ldots, m)$ such that

$$
\begin{array}{r}
\left\langle x_{i}-x_{i}^{*}, \xi_{i}^{*}+\nabla g_{i}\left(x_{i}^{*}\right)-A_{i}^{T} \lambda^{*}\right\rangle \geq 0, \\
\left\langle\lambda-\lambda^{*}, \sum_{i=1}^{m} A_{i} x_{i}^{*}-b\right\rangle \geq 0,
\end{array}
$$

for all $\left(x_{1}, x_{2}, \ldots, x_{m}, \lambda\right) \in \mathscr{W}$

The Lagrange function of (1) is given by

$$
\begin{array}{r}
L\left(x_{1}, x_{2}, \ldots, x_{m}, \lambda\right)=\sum_{i=1}^{m} \Theta_{i}\left(x_{i}\right)-\lambda^{T}\left(\sum_{i=1}^{m} A_{i} x_{i}-b\right), \\
\left(x_{1}, x_{2}, \ldots, x_{m}, \lambda\right) \in \mathscr{W} .
\end{array}
$$

Let $\left(x_{1}^{*}, x_{2}^{*}, \ldots, x_{m}^{*}, \lambda^{*}\right)$ be a saddle point of the Lagrange function $L\left(x_{1}, x_{2}, \ldots, x_{m}, \lambda\right)$. That is, for any $\lambda \in \mathscr{R}^{l}$ and $x_{i} \in \mathscr{X}_{i}(i=1,2, \ldots, m)$,

$L\left(x_{1}^{*}, x_{2}^{*}, \ldots, x_{m}^{*}, \lambda\right) \leq L\left(x_{1}^{*}, x_{2}^{*}, \ldots, x_{m}^{*}, \lambda^{*}\right) \leq L\left(x_{1}, x_{2}, \ldots, x_{m}, \lambda^{*}\right)$.

Finding a saddle point of $L\left(x_{1}, x_{2}, \ldots, x_{m}, \lambda\right)$ is equivalent to finding a $w^{*}=\left(x_{1}^{*}, x_{2}^{*}, \ldots, x_{m}^{*}, \lambda^{*}\right) \in \mathscr{W}$ such that

$$
\left\{\begin{array}{l}
\Theta_{1}\left(x_{1}\right)-\Theta_{1}\left(x_{1}^{*}\right)+\left(x_{1}-x_{1}^{*}\right)^{T}\left(-A_{1}^{T} \lambda^{*}\right) \geq 0, \quad \forall x_{1} \in X_{1}, \\
\Theta_{2}\left(x_{2}\right)-\Theta_{2}\left(x_{2}^{*}\right)+\left(x_{2}-x_{2}^{*}\right)^{T}\left(-A_{2}^{T} \lambda^{*}\right) \geq 0, \quad \forall x_{2} \in \mathscr{X}_{2}, \\
\cdots \\
\Theta_{m}\left(x_{m}\right)-\Theta_{m}\left(x_{m}^{*}\right)+\left(x_{m}-x_{m}^{*}\right)^{T}\left(-A_{m}^{T} \lambda^{*}\right) \geq 0, \quad \forall x_{m} \in \mathscr{X}_{m} \\
\left(\lambda-\lambda^{*}\right)^{T}\left(\sum_{i=1}^{m} A_{i} x_{i}^{*}-b\right) \geq 0, \quad \forall \lambda \in \mathscr{R}^{l} .
\end{array}\right.
$$

Let $\quad x=\left(x_{1}, x_{2}, \ldots, x_{m}\right)^{T}, w=\left(x_{1}, x_{2}, \ldots, x_{m}\right.$, $\lambda)^{T}, \Theta(x)=\sum_{i=1}^{m} \Theta_{i}\left(x_{i}\right)$, and

$$
G(w)=\left(-A_{1}^{T} \lambda,-A_{2}^{T} \lambda, \ldots,-A_{m}^{T} \lambda, \sum_{i=1}^{m} A_{i} x_{i}-b\right)^{T} .
$$

Then, (14) can be rewritten as the following variational inequality (VI): we obtain $w^{*}=\left(x_{1}^{*}, x_{2}^{*}, \ldots, x_{m}^{*}, \lambda^{*}\right) \in \mathscr{W}$ such that

$$
\begin{array}{r}
\operatorname{VI}(\mathscr{W}, G, \Theta): \Theta(x)-\Theta\left(x^{*}\right)+\left(w-w^{*}\right)^{T} G\left(w^{*}\right) \geq 0, \\
\forall w \in \mathscr{W} .
\end{array}
$$

Let $\mathscr{W}^{*}$ be the solution set of $\operatorname{VI}(\mathscr{W}, G, \Theta)$. Since we have assumed that the solution set of (1) is nonempty, $\mathscr{W}^{*}$ is also nonempty. It follows from the definition of $G(w)$ that

$$
\left(w^{\prime}-w^{\prime \prime}\right)^{T} G\left(w^{\prime}\right)=\left(w^{\prime}-w^{\prime \prime}\right)^{T} G\left(w^{\prime \prime}\right), \quad \forall w^{\prime}, w^{\prime \prime} \in \mathscr{W} .
$$

2.2. Some Notations. Let $x^{k}=\left(x_{1}^{k}, x_{2}^{k}, \ldots, x_{m}^{k}\right)^{T}, \quad \bar{x}^{k}=$ $\left(\bar{x}_{1}^{k}, \bar{x}_{2}^{k}, \ldots, \bar{x}_{m}^{k}\right)^{T}, \quad w^{k}=\left(x_{1}^{k}, \ldots, x_{m}^{k}, \lambda^{k}\right)^{T}, \quad \bar{w}^{k}=\left(\bar{x}_{1}^{k}, \ldots\right.$, $\left.\bar{x}_{m}^{k}, \bar{\lambda}^{k}\right)^{T}$, and $v=\left(x_{2}, \ldots, x_{m}, \lambda\right)^{T}$. Let $H$ and $G_{i}$ $(i=1,2, \ldots, m)$ be given positive definite matrices, $A=\left(A_{1}, A_{2}, \ldots, A_{m}\right) . \lambda_{\max }(\cdot)$ denotes the maximum eigenvalue of one matrix, and $\lambda_{\min }(\cdot)$ denotes the minimum eigenvalue of one matrix. The following notions will be used in the later analysis: 


$$
\begin{aligned}
& M=\left(\begin{array}{cccc}
A_{2}^{T} H A_{2} & 0 & \cdots & 0 \\
A_{3}^{T} H A_{2} & A_{3}^{T} H A_{3} & \cdots & 0 \\
\vdots & \vdots & \ddots & \vdots \\
A_{m}^{T} H A_{2} & A_{m}^{T} H A_{3} & \cdots & A_{m}^{T} H A_{m}
\end{array}\right) \text {, } \\
& \bar{M}=\left(\begin{array}{ccccc}
A_{2}^{T} H A_{2} & 0 & \cdots & 0 & 0 \\
A_{3}^{T} H A_{2} & A_{3}^{T} H A_{3} & \cdots & 0 & 0 \\
\vdots & \vdots & \ddots & \vdots & \vdots \\
A_{m}^{T} H A_{2} & A_{m}^{T} H A_{3} & \cdots & A_{m}^{T} H A_{m} & 0 \\
0 & 0 & \cdots & 0 & H^{-1}
\end{array}\right), \\
& Q=\left(\begin{array}{ccccc}
A_{2}^{T} H A_{2} & A_{2}^{T} H A_{3} & \cdots & A_{2}^{T} H A_{m} & A_{2}^{T} \\
A_{3}^{T} H A_{2} & A_{3}^{T} H A_{3} & \cdots & A_{3}^{T} H A_{m} & A_{3}^{T} \\
\vdots & \vdots & \ddots & \vdots & \vdots \\
A_{m}^{T} H A_{2} & A_{m}^{T} H A_{3} & \cdots & A_{m}^{T} H A_{m} & A_{m}^{T} \\
A_{2} & A_{3} & \cdots & A_{m} & H^{-1}
\end{array}\right), \\
& P=\operatorname{diag}\left(A_{2}^{T} H A_{2}, A_{3}^{T} H A_{3}, \ldots, A_{m}^{T} H A_{m}, H^{-1}\right) \text {, } \\
& D_{k}=\left(\begin{array}{c}
G_{1}\left(x_{1}^{k}-\bar{x}_{1}^{k}\right)+\nabla g_{1}\left(\bar{x}_{1}^{k}\right)-\nabla g_{1}\left(x_{1}^{k}\right) \\
\ldots \\
G_{i}\left(x_{i}^{k}-\bar{x}_{i}^{k}\right)+\nabla g_{i}\left(\bar{x}_{i}^{k}\right)-\nabla g_{i}\left(x_{i}^{k}\right)+A_{i}^{T} H \sum_{j=2}^{i} A_{j}\left(x_{j}^{k}-\bar{x}_{j}^{k}\right) \\
\ldots \\
G_{m}\left(x_{m}^{k}-\bar{x}_{m}^{k}\right)+\nabla g_{m}\left(\bar{x}_{m}^{k}\right)-\nabla g_{m}\left(x_{m}^{k}\right)+A_{m}^{T} H \sum_{j=2}^{m} A_{j}\left(x_{j}^{k}-\bar{x}_{j}^{k}\right) \\
H^{-1}\left(\lambda^{k}-\bar{\lambda}^{k}\right)
\end{array}\right), \\
& b_{k}=\left(w^{k}-\bar{w}^{k}\right)^{T} D_{k}+\left(\lambda^{k}-\bar{\lambda}^{k}\right)^{T} \sum_{j=2}^{m} A_{j}\left(x_{j}^{k}-\bar{x}_{j}^{k}\right) .
\end{aligned}
$$

It is easy to see that

$$
Q=\left(\begin{array}{c}
A_{2}^{T} H^{1 / 2} \\
A_{3}^{T} H^{1 / 2} \\
\vdots \\
A_{m}^{T} H^{1 / 2} \\
H^{-1 / 2}
\end{array}\right)\left(A_{2}^{T} H^{\frac{1}{2}}, A_{3}^{T} H^{\frac{1}{2}}, \ldots, A_{m}^{T} H^{\frac{1}{2}}, H^{-\frac{1}{2}}\right) \geqslant 0 .
$$

\section{Algorithm and Convergence Analysis}

In this section, we first describe G-ADMM-S and then prove its convergence via the analytic framework of the contractive-type method [29]. Throughout this section, we assume that $\lambda_{\text {min }}\left(G_{i}\right)>L_{i}(i=1,2, \ldots, m)$. We propose the iterative scheme of G-ADMM-S for solving (1) in Algorithm G-ADMM-S:
Let $\gamma \in(0,2) D_{k}$ and $b_{k}$ be defined in (18) and (19), respectively. Start with $w^{0}$. With the given iterate $w^{k}$, the new iterate $w^{k+1}$ is given as follows:

Step 1 (G-ADMM procedure). Execute scheme (8) to generate $\bar{w}^{k}$.

Step 2 (substitution procedure). Generate the new iterate $w^{k+1}$ via

$$
w^{k+1}=w^{k}-\alpha^{k} D_{k}
$$

where

$$
\alpha^{k}=\gamma \alpha_{k} \quad \text { with } \quad \alpha_{k}=\frac{b_{k}}{\left\|D_{k}\right\|^{2}}
$$

Next, we establish the global convergence of Algorithm G-ADMM-S following the analytic framework of contractive-type methods. We outline the proof sketch as follows:

(1) Prove that $-D_{k}$ is a descent direction of the function $(1 / 2)\left\|w-w^{*}\right\|^{2}$ at the point $w=w^{k}$ whenever 
$w^{k} \neq \bar{w}^{k}$, where $\bar{w}^{k}$ is generated by G-ADMM scheme (8) and $w^{*} \in \mathscr{W}^{*}$

(2) Prove that the sequence generated by Algorithm G-ADMM-S is contractive with respect to $\mathscr{W}^{*}$

(3) Establish the convergence

Accordingly, we divide the convergence analysis into three sections to address the claims listed above.

3.1. Verification of the Descent Direction. In this section, we show that $-D_{k}$ is a descent direction of the function $(1 / 2)\left\|w-w^{*}\right\|^{2}$ at the point $w=w^{k}$ whenever $w^{k} \neq \bar{w}^{k}$ and $w^{*} \in \mathscr{W}^{*}$. For this purpose, we first prove an important inequality for the output of G-ADMM procedure (8), which will be used often in our further discussion.

Theorem 1. $\bar{w}^{k} \in \mathscr{W}$ and

$$
\Theta(x)-\Theta\left(\bar{x}^{k}\right)+\left(w-\bar{w}^{k}\right)^{T} G\left(\bar{w}^{k}\right)+P_{k} \geq\left(w-\bar{w}^{k}\right)^{T} D_{k},
$$$$
\text { where } P_{k}=\left(w-\bar{w}^{k}\right)^{T}\left(\begin{array}{c}
A_{1}^{T} \\
A_{2}^{T} \\
\vdots \\
A_{m}^{T} \\
0
\end{array}\right) H \sum_{j=2}^{m} A_{j}\left(x_{j}^{k}-\bar{x}_{j}^{k}\right) \text {. }
$$

Proof. By the optimality condition of the $x_{i}$-related subproblem in (8), for $i=1,2, \ldots, m$, we have $\bar{x}_{i}^{k} \in X_{i}$ and

$$
\begin{aligned}
0 & \in \partial f_{i}\left(\bar{x}_{i}^{k}\right)+\nabla g_{i}\left(x_{i}^{k}\right)+A_{i}^{T} H\left(\sum_{j=1}^{i} A_{j} \bar{x}_{j}^{k}+\sum_{j=i+1}^{m} A_{j} x_{j}^{k}-b-H^{-1} \lambda^{k}\right) \\
& +G_{i}\left(\bar{x}_{i}^{k}-x_{i}^{k}\right)+\partial \delta\left(\bar{x}_{i}^{k} \mid x_{i}\right),
\end{aligned}
$$

where $\delta\left(\mathscr{X}_{i}\right)$ is the indicator function of the set $\mathscr{X}_{i}$. Thus, $\bar{w}^{k} \in \mathscr{W}$ and there exists $\eta \in \partial \delta\left(X_{i}\right)\left(\bar{x}_{i}^{k}\right)$ such that

$$
-\eta \in \partial f_{i}\left(\bar{x}_{i}^{k}\right)+\nabla g_{i}\left(\bar{x}_{i}^{k}\right)+\rho=\partial \Theta_{i}\left(\bar{x}_{i}^{k}\right)+\rho,
$$

where $\rho:=\nabla g_{i}\left(x_{i}^{k}\right)-\nabla g_{i}\left(\bar{x}_{i}^{k}\right)+A_{i}^{T} H\left(\sum_{j=1}^{i} A_{j} \bar{x}_{j}^{k}+\sum_{j=i+1}^{m}\right.$ $\left.A_{j} x_{j}^{k}-b-H^{-1} \lambda^{k}\right)+G_{i}\left(\bar{x}_{i}^{k}-x_{i}^{k}\right)$. From the subgradient inequality, one has

$$
\begin{array}{rr}
\Theta_{i}\left(x_{i}\right)-\Theta_{i}\left(\bar{x}_{i}^{k}\right) \geq\left(x_{i}-\bar{x}_{i}^{k}\right)^{T}(-\eta-\rho), & \forall x_{i} \in X_{i}, \\
\Theta_{i}\left(x_{i}\right)-\Theta_{i}\left(\bar{x}_{i}^{k}\right)+\left(x_{i}-\bar{x}_{i}^{k}\right)^{T} \rho \geq-\left(x_{i}-\bar{x}_{i}^{k}\right)^{T} \eta, & \forall x_{i} \in X_{i} .
\end{array}
$$

From the definition of $\partial \delta\left(\mathscr{X}_{i}\right)\left(\bar{x}_{i}^{k}\right)$, one has

$$
\Theta_{i}\left(x_{i}\right)-\Theta_{i}\left(\bar{x}_{i}^{k}\right)+\left(x_{i}-\bar{x}_{i}^{k}\right)^{T} \rho \geq 0, \quad \forall x_{i} \in \mathscr{X}_{i} .
$$

That is,

$$
\begin{aligned}
0 \leq & \Theta_{i}\left(x_{i}\right)-\Theta_{i}\left(\bar{x}_{i}^{k}\right)+\left(x_{i}-\bar{x}_{i}^{k}\right)^{T}\left\{\nabla g_{i}\left(x_{i}^{k}\right)\right. \\
& -\nabla g_{i}\left(\bar{x}_{i}^{k}\right)+A_{i}^{T} H\left(\sum_{j=1}^{i} A_{j} \bar{x}_{j}^{k}\right. \\
& \left.\left.+\sum_{j=i+1}^{m} A_{j} x_{j}^{k}-b-H^{-1} \lambda^{k}\right)+G_{i}\left(\bar{x}_{i}^{k}-x_{i}^{k}\right)\right\},
\end{aligned}
$$

for all $x_{i} \in \mathscr{X}_{i}$. Substituting $\bar{\lambda}^{k}=\lambda^{k}-H\left(\sum_{j=1}^{m} A_{j} \bar{x}_{j}^{k}-b\right)$ (see (8)) in the above inequality, we obtain

$$
\begin{array}{r}
0 \leq \Theta_{i}\left(x_{i}\right)-\Theta_{i}\left(\bar{x}_{i}^{k}\right)+\left(x_{i}-\bar{x}_{i}^{k}\right)^{T}\left\{\nabla g_{i}\left(x_{i}^{k}\right)-\nabla g_{i}\left(\bar{x}_{i}^{k}\right)\right. \\
\left.-A_{i}^{T} \bar{\lambda}^{k}+A_{i}^{T} H \sum_{j=i+1}^{m} A_{j}\left(x_{j}^{k}-\bar{x}_{j}^{k}\right)+G_{i}\left(\bar{x}_{i}^{k}-x_{i}^{k}\right)\right\},
\end{array}
$$

for all $x_{i} \in \mathscr{X}_{i}$. Summing the above inequality over $i=1,2, \ldots, m$, we obtain

$$
\Theta(x)-\Theta\left(\bar{x}^{k}\right)+\left(x-\bar{x}^{k}\right)^{T}\left(-A^{T} \bar{\lambda}^{k}\right) C_{k} \geq 0,
$$

where

$$
C_{k}=\left(\begin{array}{c}
G_{1}\left(\bar{x}_{1}^{k}-x_{1}^{k}\right)+\nabla g_{1}\left(x_{1}^{k}\right)-\nabla g_{1}\left(\bar{x}_{1}^{k}\right)+A_{1}^{T} H \sum_{j=2}^{m} A_{j}\left(x_{j}^{k}-\bar{x}_{j}^{k}\right) \\
\ldots \\
G_{i}\left(\bar{x}_{i}^{k}-x_{i}^{k}\right)+\nabla g_{i}\left(x_{i}^{k}\right)-\nabla g_{i}\left(\bar{x}_{i}^{k}\right)+A_{i}^{T} H \sum_{j=i+1}^{m} A_{j}\left(x_{j}^{k}-\bar{x}_{j}^{k}\right) \\
\ldots \\
G_{m}\left(\bar{x}_{m}^{k}-x_{m}^{k}\right)+\nabla g_{m}\left(x_{m}^{k}\right)-\nabla g_{m}\left(\bar{x}_{m}^{k}\right)
\end{array}\right)
$$


Then, by adding the following term

$$
\left(x-\bar{x}^{k}\right)^{T}\left(\begin{array}{c}
G_{1}\left(x_{1}^{k}-\bar{x}_{1}^{k}\right)+\nabla g_{1}\left(\bar{x}_{1}^{k}\right)-\nabla g_{1}\left(x_{1}^{k}\right) \\
\ldots \\
G_{i}\left(x_{i}^{k}-\bar{x}_{i}^{k}\right)+\nabla g_{i}\left(\bar{x}_{i}^{k}\right)-\nabla g_{i}\left(x_{i}^{k}\right)+A_{i}^{T} H \sum_{j=2}^{i} A_{j}\left(x_{j}^{k}-\bar{x}_{j}^{k}\right) \\
\ldots \\
G_{m}\left(x_{m}^{k}-\bar{x}_{m}^{k}\right)+\nabla g_{m}\left(\bar{x}_{m}^{k}\right)-\nabla g_{m}\left(x_{m}^{k}\right)+A_{m}^{T} H \sum_{j=2}^{m} A_{j}\left(x_{j}^{k}-\bar{x}_{j}^{k}\right)
\end{array}\right)
$$

to both sides of (30), we get

$$
\left.\begin{array}{c}
\Theta(x)-\Theta\left(\bar{x}^{k}\right)+\left(x-\bar{x}^{k}\right)^{T}\left\{\left(-A^{T} \bar{\lambda}^{k}\right)+\left(\begin{array}{c}
A_{1}^{T} H \sum_{j=2}^{m} A_{j}\left(x_{j}^{k}-\bar{x}_{j}^{k}\right) \\
\ldots \\
A_{i}^{T} H \sum_{j=2}^{m} A_{j}\left(x_{j}^{k}-\bar{x}_{j}^{k}\right) \\
\cdots \\
A_{m}^{T} H \sum_{j=2}^{m} A_{j}\left(x_{j}^{k}-\bar{x}_{j}^{k}\right)
\end{array}\right)\right\} \geq \\
G_{1}\left(x_{1}^{k}-\bar{x}_{1}^{k}\right)+\nabla g_{1}\left(\bar{x}_{1}^{k}\right)-\nabla g_{1}\left(x_{1}^{k}\right) \\
\ldots \\
G_{i}\left(x_{i}^{k}-\bar{x}_{i}^{k}\right)+\nabla g_{i}\left(\bar{x}_{i}^{k}\right)-\nabla g_{i}\left(x_{i}^{k}\right)+A_{i}^{T} H \sum_{j=2}^{i} A_{j}\left(x_{j}^{k}-\bar{x}_{j}^{k}\right) \\
\ldots \\
G_{m}\left(x_{m}^{k}-\bar{x}_{m}^{k}\right)+\nabla g_{m}\left(\bar{x}_{m}^{k}\right)-\nabla g_{m}\left(x_{m}^{k}\right)+A_{m}^{T} H \sum_{j=2}^{m} A_{j}\left(x_{j}^{k}-\bar{x}_{j}^{k}\right)
\end{array}\right) .
$$

Since $\bar{\lambda}^{k}=\lambda^{k}-H\left(\sum_{j=1}^{m} A_{j} \bar{x}_{j}^{k}-b\right)$, we have

$$
\left(\sum_{j=1}^{m} A_{j} \bar{x}_{j}^{k}-b\right)+H^{-1}\left(\bar{\lambda}^{k}-\lambda^{k}\right)=0 .
$$

$$
\Theta(x)-\Theta\left(\bar{x}^{k}\right)+\left(w-\bar{w}^{k}\right)^{T}\left(G\left(\bar{w}^{k}\right)+H_{k}\right) \geq\left(w-\bar{w}^{k}\right)^{T} Q_{k},
$$

where

Combining the above two formulas, we have

$$
\begin{aligned}
& H_{k}=\left(A_{1}^{T} H \sum_{j=2}^{m} A_{j}\left(x_{j}^{k}-\bar{x}_{j}^{k}\right), A_{2}^{T} H \sum_{j=2}^{m} A_{j}\left(x_{j}^{k}-\bar{x}_{j}^{k}\right), \ldots, A_{m}^{T} H \sum_{j=2}^{m} A_{j}\left(x_{j}^{k}-\bar{x}_{j}^{k}\right), 0\right)^{T}, \\
& Q_{k}=\left(\begin{array}{c}
G_{1}\left(x_{1}^{k}-\bar{x}_{1}^{k}\right)+\nabla g_{1}\left(\bar{x}_{1}^{k}\right)-\nabla g_{1}\left(x_{1}^{k}\right) \\
\ldots \\
G_{i}\left(x_{i}^{k}-\bar{x}_{i}^{k}\right)+\nabla g_{i}\left(\bar{x}_{i}^{k}\right)-\nabla g_{i}\left(x_{i}^{k}\right)+A_{i}^{T} H \sum_{j=2}^{i} A_{j}\left(x_{j}^{k}-\bar{x}_{j}^{k}\right) \\
\ldots \\
G_{m}\left(x_{m}^{k}-\bar{x}_{m}^{k}\right)+\nabla g_{m}\left(\bar{x}_{m}^{k}\right)-\nabla g_{m}\left(x_{m}^{k}\right)+A_{m}^{T} H \sum_{j=2}^{m} A_{j}\left(x_{j}^{k}-\bar{x}_{j}^{k}\right) \\
H^{-1}\left(\lambda^{k}-\bar{\lambda}^{k}\right)
\end{array}\right) .
\end{aligned}
$$


Using the notations of $G\left(\bar{w}^{k}\right)$ (see (15)) and $D_{k}$ (see $(18)$ ), assertion (23) is proved.

Based on assertion (23), we can get the following result.

\section{Corollary 1.}

$$
\left(\bar{w}^{k}-w^{*}\right)^{T} D_{k} \geq\left(\lambda^{k}-\bar{\lambda}^{k}\right)^{T} \sum_{j=2}^{m} A_{j}\left(x_{j}^{k}-\bar{x}_{j}^{k}\right), \quad \forall w^{*} \in \mathscr{W}^{*} .
$$

Proof. It follows from (23) that

$$
\begin{aligned}
\left(\bar{w}^{k}-w^{*}\right)^{T} D_{k} \geq & \Theta\left(\bar{x}^{k}\right)-\Theta\left(x^{*}\right)+\left(\bar{w}^{k}-w^{*}\right)^{T} G\left(\bar{w}^{k}\right) \\
& +\left(\bar{w}^{k}-w^{*}\right)^{T}\left(\begin{array}{c}
A_{1}^{T} \\
A_{2}^{T} \\
\vdots \\
A_{m}^{T}
\end{array}\right) H \sum_{j=2}^{m} A_{j}\left(x_{j}^{k}-\bar{x}_{j}^{k}\right) .
\end{aligned}
$$

Using (17) and the optimality of $w^{*}$, we have

$$
\begin{aligned}
& \Theta\left(\bar{x}^{k}\right)-\Theta\left(x^{*}\right)+\left(\bar{w}^{k}-w^{*}\right)^{T} G\left(\bar{w}^{k}\right)=\Theta\left(\bar{x}^{k}\right)-\Theta\left(x^{*}\right) \\
& \quad+\left(\bar{w}^{k}-w^{*}\right)^{T} G\left(w^{*}\right) \geq 0 .
\end{aligned}
$$

Thus,

$$
\left(\bar{w}^{k}-w^{*}\right)^{T} D_{k} \geq\left[H\left(\sum_{j=1}^{m} A_{j} \bar{x}_{j}^{k}-\sum_{j=1}^{m} A_{j} x_{j}^{*}\right)\right]^{T} \sum_{j=2}^{m} A_{j}\left(x_{j}^{k}-\bar{x}_{j}^{k}\right) .
$$

Since $\sum_{j=1}^{m} A_{j} x_{j}^{*}=b$ and $H\left(\sum_{j=1}^{m}\left(A_{j} \bar{x}_{j}^{k}-b\right)\right)=\lambda^{k}-\bar{\lambda}^{k}$,

$$
\left(\bar{w}^{k}-w^{*}\right)^{T} D_{k} \geq\left(\lambda^{k}-\bar{\lambda}^{k}\right)^{T} \sum_{j=2}^{m} A_{j}\left(x_{j}^{k}-\bar{x}_{j}^{k}\right) \text {. }
$$

The next theorem implies that $-D_{k}$ is a descent direction of the function $(1 / 2)\left\|w-w^{*}\right\|^{2}$ at the point $w=w^{k}$ whenever $w^{k} \neq \bar{w}^{k}$.

Theorem 2. For all $w^{*} \in \mathscr{W}^{*}$,

$$
\begin{aligned}
\left(w^{k}-w^{*}\right)^{T} D_{k} & \geq b_{k} \\
& \geq \sum_{j=1}^{m}\left(\left\|x_{j}^{k}-\bar{x}_{j}^{k}\right\|_{G_{j}}^{2}-L_{i}\left\|x_{j}^{k}-\bar{x}_{j}^{k}\right\|\right)+\frac{1}{2}\left\|v^{k}-\bar{v}^{k}\right\|_{(P+Q)}^{2} \\
& \geq \sum_{j=1}^{m}\left(\lambda_{\min }\left(G_{j}\right)-L_{i}\right)\left\|x_{j}^{k}-\bar{x}_{j}^{k}\right\|^{2}+\frac{1}{2}\left\|\lambda^{k}-\bar{\lambda}^{k}\right\|_{H^{-1}}^{2} .
\end{aligned}
$$

Proof. It follows from (37) that

$$
\left(w^{k}-w^{*}\right)^{T} D_{k} \geq\left(w^{k}-\bar{w}^{k}\right)^{T} D_{k}+\left(\lambda^{k}-\bar{\lambda}^{k}\right)^{T} \sum_{j=2}^{m} A_{j}\left(x_{j}^{k}-\bar{x}_{j}^{k}\right) .
$$

That is, $\left(w^{k}-w^{*}\right)^{T} D_{k} \geq b_{k}$. Now, we treat the first term of the right-hand side of (43):

$$
\begin{aligned}
\left(w^{k}-\bar{w}^{k}\right)^{T} D_{k}= & \sum_{i=1}^{m}\left[\left\|x_{i}^{k}-\bar{x}_{i}^{k}\right\|_{G_{i}}^{2}+\left(x_{i}^{k}-\bar{x}_{i}^{k}\right)^{T}\left(\nabla g_{i}\left(\bar{x}_{i}^{k}\right)-\nabla g_{i}\left(x_{i}^{k}\right)\right)\right] \\
& +\left(v^{k}-\bar{v}^{k}\right)^{T} \bar{M}\left(v^{k}-\bar{v}^{k}\right) \\
\geq & \sum_{i=1}^{m}\left(\left\|x_{i}^{k}-\bar{x}_{i}^{k}\right\|_{G_{i}}^{2}-L_{i}\left\|x_{j}^{k}-\bar{x}_{j}^{k}\right\|\right)+\left(v^{k}-\bar{v}^{k}\right)^{T} \bar{M}\left(v^{k}-\bar{v}^{k}\right) \\
\geq & \sum_{i=1}^{m}\left(\lambda_{\min }\left(G_{i}\right)-L_{i}\right)\left\|x_{i}^{k}-\bar{x}_{i}^{k}\right\|^{2}+\left(v^{k}-\bar{v}^{k}\right)^{T} \bar{M}\left(v^{k}-\bar{v}^{k}\right),
\end{aligned}
$$

where the first inequality follows from the Lipschitz continuous of $\nabla g_{i}$. Then, let us deal with the second term of the right-hand side of (43):

$$
\left(\lambda^{k}-\bar{\lambda}^{k}\right)^{T} \sum_{j=2}^{m} A_{j}\left(x_{j}^{k}-\bar{x}_{j}^{k}\right)=\left(v^{k}-\bar{v}^{k}\right)^{T}\left(\begin{array}{ccccc}
0 & 0 & \cdots & 0 & 0 \\
0 & 0 & \cdots & 0 & 0 \\
\vdots & \vdots & \ddots & \vdots & \vdots \\
0 & 0 & \cdots & 0 & 0 \\
A_{2} & A_{3} & \cdots & A_{m} & 0
\end{array}\right)\left(v^{k}-\bar{v}^{k}\right)
$$


Thus,

$$
\begin{aligned}
& \left(v^{k}-\bar{v}^{k}\right)^{T} \bar{M}\left(v^{k}-\bar{v}^{k}\right)+\left(\lambda^{k}-\bar{\lambda}^{k}\right)^{T} \sum_{j=2}^{m} A_{j}\left(x_{j}^{k}-\bar{x}_{j}^{k}\right) \\
& =\left(v^{k}-\bar{v}^{k}\right)^{T} M_{1}\left(v^{k}-\bar{v}^{k}\right) \\
& =\frac{1}{2}\left(v^{k}-\bar{v}^{k}\right)^{T} M_{2}\left(v^{k}-\bar{v}^{k}\right) \\
& =\frac{1}{2}\left\|v^{k}-\bar{v}^{k}\right\|_{P}^{2}+\frac{1}{2}\left\|v^{k}-\bar{v}^{k}\right\|_{Q}^{2} \\
& \geq \frac{1}{2}\left\|\lambda^{k}-\bar{\lambda}^{k}\right\|_{H^{-1}}^{2},
\end{aligned}
$$

where

$$
\begin{aligned}
M_{1}= & \left(\begin{array}{ccccc}
A_{2}^{T} H A_{2} & 0 & \cdots & 0 & 0 \\
A_{3}^{T} H A_{2} & A_{3}^{T} H A_{3} & \cdots & 0 & 0 \\
\vdots & \vdots & \ddots & \vdots & \vdots \\
A_{m}^{T} H A_{2} & A_{m}^{T} H A_{3} & \cdots & A_{m}^{T} H A_{m} & 0 \\
A_{2} & A_{3} & \cdots & A_{m} & H^{-1}
\end{array}\right), \\
M_{2}= & \left(\begin{array}{ccccc}
2 A_{2}^{T} H A_{2} & A_{2}^{T} H A_{3} & \cdots & A_{2}^{T} H A_{m} & A_{2}^{k} \\
A_{3}^{T} H A_{2} & 2 A_{3}^{T} H A_{3} & \cdots & A_{3}^{T} H A_{m} & A_{3}^{k} \\
\vdots & \vdots & \ddots & \vdots & \vdots \\
A_{m}^{T} H A_{2} & A_{m}^{T} H A_{3} & \cdots & 2 A_{m}^{T} H A_{m} & A_{m}^{k} \\
A_{2} & A_{3} & \cdots & A_{m} & 2 H^{-1}
\end{array}\right) .
\end{aligned}
$$

The assertion follows from the above three formulas.

Since $H>0$ and $\lambda_{\min }\left(G_{i}\right)>L_{i}$, whenever $w^{k}-\bar{w}^{k} \neq 0$, assertion (42) shows the positivity of the term $\left(w^{k}-w^{*}\right)^{T} D_{k}$, and thus, the direction $-D_{k}$ is a descent direction of the function $(1 / 2)\left\|w-w^{*}\right\|^{2}$ at the point $w=w^{k}$

3.2. Contractive Property. In this section, we show that the sequence $\left\{w^{k}\right\}$ generated by Algorithm G-ADMM-S is contractive with respect to the set $\mathscr{W}^{*}$.

Since the direction $-D_{k}$ is a descent direction of the function $(1 / 2)\left\|w-w^{*}\right\|^{2}$ at the point $w=w^{k}$, the new iterate $w^{k+1}$ can be generated by

$$
w^{k+1}=w^{k}-\alpha D_{k}
$$

Thus,

$$
\begin{aligned}
\left\|w^{k}-w^{*}\right\|^{2}-\left\|w^{k+1}-w^{*}\right\|^{2} & =\left\|w^{k}-w^{*}\right\|^{2}-\left\|\left(w^{k}-w^{*}\right)-\alpha D_{k}\right\|^{2} \\
& =2 \alpha\left(w^{k}-w^{*}\right)^{T} D_{k}-\alpha^{2}\left\|D_{k}\right\|^{2} \\
& \geq 2 \alpha b_{k}-\alpha^{2}\left\|D_{k}\right\|^{2}, \quad \forall w^{*} \in \mathscr{W}^{*}
\end{aligned}
$$

where the inequality follows from the first inequality of (42).
Let $q(\alpha)=2 \alpha b_{k}-\alpha^{2}\left\|D_{k}\right\|^{2}$. Note that right-hand side of (49), i.e., $q(\alpha)$, is a quadratic function of $\alpha$, and

$$
q(\alpha)>0 \Longleftrightarrow 0<\alpha<\frac{2 b_{k}}{\left\|D_{k}\right\|^{2}} \text {. }
$$

In order to obtain the closest proximity to $\mathscr{W}^{*}$, we are in the desire to maximize this quadratic function and this promotes us to take the optimal value of $\alpha$ as

$$
\alpha=\alpha_{k}:=\frac{b_{k}}{\left\|D_{k}\right\|^{2}} \text {. }
$$

With this choice of step size, it follows from (49) that

$$
\left\|w^{k}-w^{*}\right\|^{2} \leq\left\|w^{k}-w^{*}\right\|^{2}-\frac{b_{k}^{2}}{\left\|D_{k}\right\|^{2}}, \quad \forall w^{*} \in \mathscr{W}^{*} .
$$

Let

$$
\begin{aligned}
T_{1}= & \max \left\{\lambda_{\max }\left(H^{-1}\right), \lambda_{\max }\left(G_{i}\right)+L_{i}, 1 \leq i \leq m\right\} \\
& +\sqrt{\lambda_{\max }\left(M^{T} M\right)}>0, \\
T_{2}= & \min \left\{\frac{\lambda_{\min }\left(H^{-1}\right)}{2}, \lambda_{\min }\left(G_{i}\right)-L_{i}, 1 \leq i \leq m\right\}>0 .
\end{aligned}
$$

From the definition of $D_{k}$ (see, (18)), one has

$$
\begin{aligned}
\left\|D_{k}\right\| \leq & \left\|\bar{G}_{k}\right\|+\left\|M\left(v^{k}-\bar{v}^{k}\right)\right\| \\
\leq & {\left[\max \left\{\lambda_{\max }\left(H^{-1}\right), \lambda_{\max }\left(G_{i}\right)+L_{i}, 1 \leq i \leq m\right\}\right.} \\
& \left.+\sqrt{\lambda_{\max }\left(M^{T} M\right)}\right]\left\|w^{k}-\bar{w}^{k}\right\| \\
\leq & T_{1}\left\|w^{k}-\bar{w}^{k}\right\|,
\end{aligned}
$$

where

$$
\bar{G}_{k}=\left(\begin{array}{c}
G_{1}\left(x_{1}^{k}-\bar{x}_{1}^{k}\right)+\nabla g_{1}\left(\bar{x}_{1}^{k}\right)-\nabla g_{1}\left(x_{1}^{k}\right) \\
G_{2}\left(x_{2}^{k}-\bar{x}_{2}^{k}\right)+\nabla g_{2}\left(\bar{x}_{2}^{k}\right)-\nabla g_{2}\left(x_{2}^{k}\right) \\
\ldots \\
G_{m}\left(x_{m}^{k}-\bar{x}_{m}^{k}\right)+\nabla g_{m}\left(\bar{x}_{m}^{k}\right)-\nabla g_{m}\left(x_{m}^{k}\right) \\
H^{-1}\left(\lambda^{k}-\bar{\lambda}^{k}\right)
\end{array}\right) .
$$

It follows from (19) and (42) that

$$
\begin{aligned}
b_{k} & \geq \min \left\{\frac{\lambda_{\min }\left(H^{-1}\right)}{2}, \lambda_{\min }\left(G_{i}\right)-L_{i}, 1 \leq i \leq m\right\}\left\|w^{k}-\bar{w}^{k}\right\|^{2} \\
& \geq T_{2}\left\|w^{k}-\bar{w}^{k}\right\|^{2} .
\end{aligned}
$$

It is easy to see that $\alpha_{k} \geq\left(T_{2} / T_{1}^{2}\right)$.

Next, we show that the sequence $\left\{w^{k}\right\}$ generated by Algorithm G-ADMM-S is contractive with respect to the set $\mathscr{W}^{*}$. 
Theorem 3. Let the sequence $\left\{w^{k}\right\}$ be generated by the proposed Algorithm G-ADMM-S. Then,

$$
\left\|w^{k+1}-w^{*}\right\|^{2} \leq\left\|w^{k}-w^{*}\right\|^{2}-\frac{\gamma(2-\gamma) T_{2}^{2}}{T_{1}^{2}}\left\|\bar{w}^{k}-w^{k}\right\|^{2}, \quad \forall w^{*} \in \mathscr{W}^{*} .
$$

Proof. Using (21) and (22), we obtain

$$
\begin{aligned}
\left\|w^{k+1}-w^{*}\right\|^{2}= & \left\|w^{k}-w^{*}\right\|^{2}-2 \gamma \alpha_{k}\left(w^{k}-w^{*}\right)^{T} D_{k} \\
& +\left(\gamma \alpha_{k}\right)^{2}\left\|D_{k}\right\|^{2} \\
\leq & \left\|w^{k}-w^{*}\right\|^{2}-\left[2 \gamma \alpha_{k} b_{k}-\left(\gamma \alpha_{k}\right)^{2}\left\|D_{k}\right\|^{2}\right] \\
= & \left\|w^{k}-w^{*}\right\|^{2}-\gamma(2-\gamma) \alpha_{k} b_{k} \\
= & \left\|w^{k}-w^{*}\right\|^{2}-\frac{\gamma(2-\gamma) b_{k}^{2}}{\left\|D_{k}\right\|^{2}} \\
\leq & \left\|w^{k}-w^{*}\right\|^{2}-\frac{\gamma(2-\gamma) T_{2}^{2}}{T_{1}^{2}}\left\|\bar{w}^{k}-w^{k}\right\|^{2},
\end{aligned}
$$

where the first inequality follows from the first inequality of (42) and the second inequality follows from (54)-(56).

3.3. Convergence Result. In this section, we establish the global convergence for Algorithm G-ADMM-S based on the analytic framework of contraction methods in [29].

Theorem 4(global convergence). Let the sequence $\left\{w^{k}\right\}$ be generated by Algorithm G-ADMM-S. Then, there exists $w^{\infty} \in \mathscr{W}^{*}$ such that

$$
\lim _{k \longrightarrow \infty} w^{k}=w^{\infty}
$$

Proof. It follows from (57) that the sequence $\left\{w^{k}\right\}$ is bounded and

$$
\gamma(2-\gamma) \sum_{k=0}^{\infty} \frac{T_{2}^{2}}{T_{1}^{2}}\left\|\bar{w}^{k}-w^{k}\right\|^{2} \leq\left\|w^{0}-w^{*}\right\|^{2}
$$

which implies that $\lim _{k \rightarrow \infty}\left\|\bar{w}^{k}-w^{k}\right\|=0$.

Since $\left\{w^{k}\right\}$ is bounded, the sequence $\left\{w^{k}\right\}$ has at least one cluster point and we denote it by $w^{\infty}=\left(x_{1}^{\infty}, x_{2}^{\infty}, \ldots, x_{m}^{\infty}, \lambda^{\infty}\right)$. In addition, let $\left\{w^{k_{j}}\right\}$ be the subsequence converging to $w^{\infty}$. Since $\lim _{k \longrightarrow \infty}\left\|w^{k}-\bar{w}^{k}\right\|=0,\left\{\bar{w}^{k_{j}}\right\}$ converges to $w^{\infty}$.

By taking the limit over $k_{j}$ in (15), we have that

$$
\Theta(x)-\Theta\left(x^{\infty}\right)+\left(w-w^{\infty}\right) G(w) \geq 0, \quad \forall w \in \mathscr{W} .
$$

Therefore, $w^{\infty}$ is a solution point of $V I(\mathscr{W}, G, \Theta)$. By using (57), we have

$$
\left\|w^{k+1}-w^{\infty}\right\|^{2} \leq\left\|w^{k}-w^{\infty}\right\|^{2}-\frac{\gamma(2-\gamma) T_{2}^{2}}{T_{1}^{2}}\left\|\bar{w}^{k}-w^{k}\right\|^{2}
$$

and thus, $\lim _{k \rightarrow \infty} w^{k}=w^{\infty}$.

\section{Iteration Complexity}

In this section, we will show that after $t$ iterations of $\mathrm{Al}$ gorithm G-ADMM-S, we can ensure that

$$
\min _{0 \leq k \leq t}\left\{\left\|w^{k}-w^{k}\right\|^{2}\right\} \leq \varepsilon
$$

where $\varepsilon=O(1 / t)$. Thus, a worst-case $O(1 / \varepsilon)$ iteration complexity is established in nonergodic sense for Algorithm G-ADMM-S.

Theorem 5. Let the sequence $\left\{w^{k}\right\}$ be generated by Algorithm G-ADMM-S. Then,

$$
\min _{0 \leq k \leq t}\left\{\left\|\bar{w}^{k}-w^{k}\right\|^{2}\right\} \leq \frac{1}{c(t+1)}\left\|w^{0}-w^{*}\right\|^{2}, \quad \forall w^{*} \in \mathscr{W}^{*},
$$

where $c\left(\gamma(2-\gamma) T_{2}^{2} / T_{1}^{2}\right)$.

Proof. It follows from (57) that

$$
c \sum_{k=0}^{\infty}\left\|\bar{w}^{k}-w^{k}\right\|^{2} \leq\left\|w^{0}-w^{*}\right\|^{2}, \quad \forall w^{*} \in \mathscr{W}^{*} .
$$

Thus, for any integer $t>0$, we obtain

$$
c \sum_{k=0}^{t}\left\|\bar{w}^{k}-w^{k}\right\|^{2} \leq\left\|w^{0}-w^{*}\right\|^{2}, \quad \forall w^{*} \in \mathscr{W}^{*},
$$

and consequently, we obtain assertion (64).

Recall that $\mathscr{W}^{*}$ is convex and closed under our assumptions (see, Theorem 2.3.5 in [30]). Let

$$
d:=\inf \left\{\left\|w^{0}-w^{*}\right\|^{2} \mid w^{*} \in \mathscr{W}^{*}\right\} .
$$

For any given $\varepsilon>0$, inequality (64) indicates that Algorithm G-ADMM-S requires at most $\lfloor d /(c \varepsilon)\rfloor$ iterations to fulfill the requirement $\left\|\bar{w}^{k}-w^{k}\right\|^{2} \leq \varepsilon$.

\section{Numerical Results}

To investigate the numerical performance of the proposed algorithm, we apply it to solve a convex quadratic programming and a nonlinear convex programming with separable structure and report some preliminary numerical results. All codes were written by Matlab 2016a, and all the numerical experiments were conducted on a Dell desktop computer with Intel Pentium Intel (R) Core processor $3.30 \mathrm{GHz}$ and $4 \mathrm{~GB}$ memory.

5.1. Quadratic Programming Problem. First, we consider the following quadratic programming problem: 


$$
\begin{aligned}
& \min \sum_{i=1}^{3}\left(\frac{1}{2} x_{i}^{T} M_{i} x_{i}+q_{i}^{T} x_{i}\right) \\
& \text { s.t. } \quad A_{1} x_{1}+x_{2}+A_{3} x_{3}=b, \\
& \quad x_{1} \in \mathscr{R}^{n_{1}}, \quad B_{L} \leq x_{1} \leq B_{U}, \quad x_{2} \in \mathscr{R}^{n_{2}}, \\
& \left\|x_{2}\right\| \leq r, \quad x_{3} \in \mathscr{R}_{+}^{n_{3}} .
\end{aligned}
$$

In the experiments, we set $f_{i}\left(x_{i}\right)=q_{1}^{T} x_{i}$ and $g_{i}\left(x_{1}\right)=(1 / 2) x_{i}^{T} M_{i} x_{i},(i=1,2,3)$. We set the matrix $M_{1}=$ $I_{n_{1} \times n_{1}}$ and construct the rest of matrices $M_{i}(i=2,3)$ in a way similar to $[25,31]$. That is, $M_{i}=V_{i}^{T} V_{i}+\tau I_{n_{i}}$, where $V_{i}$ are random matrices and

$$
\tau=\frac{\lambda_{\max }\left(V_{i}^{T} V_{i}\right)-t \cdot \lambda_{\min }\left(V_{i}^{T} V_{i}\right)}{t-1} .
$$

In our tests, we set $t=10^{3}$ and generate the matrices $V_{i}=$ rand $\left(\left(n_{i} / 5\right), n_{i}\right)$ in Matlab function. In the experiments, we set $\left[B_{L}, B_{U}\right]=[0,10]$ and the radius $r=10$. For the linear constraint, the entries of $A_{i} \in \mathscr{R}^{l \times n_{i}}(i=1,3)$ are uniformly distributed in $(0,1)$ with the density 0.1 and $l=n_{2} . x_{i}^{*}$ 's are given generated by $x_{i}^{*}=\operatorname{sprand}\left(n_{i}, 1,0.5\right)(i=1,2,3)$ in the Matlab function. In order to guarantee the feasibility of the problem, we set $q_{i}=-M_{i} x_{i}^{*}, \quad(i=1,2,3)$ and $b=A_{1} x_{1}^{*}+x_{2}^{*}+A_{3} x_{3}^{*}$. Thus, $\left(x_{1}^{*}, x_{2}^{*}, x_{3}^{*}\right)$ is an optimal solution of (68). The Algorithm G-ADMM-S is compared with the PPSM-C in [25]. The initial iteration points are the zero vectors $x_{i}^{0}=0_{n_{i} \times 1}(i=1,2,3)$ and $\lambda^{0}=0_{l \times 1}$ for all tested algorithms. We set a maximal number of 20000 for iteration of the proposed algorithms with a modified stopping criterion as follows:

$$
\max _{i=1,2,3}\left\{\frac{\left\|x_{i}^{k}-\bar{x}_{i}^{k}\right\|}{\left\|x_{i}^{k}\right\|}, \frac{\left\|\lambda^{k}-\bar{\lambda}^{k}\right\|}{\left\|\lambda^{k}\right\|}\right\} \leq 10^{-2} .
$$

Now, we specify the choices of parameters to implement these algorithms. First, we set $H=\beta I$ with $\beta=0.01$ and the relaxation parameter $\gamma=1.8$ for all tested algorithms. For "PPSM-C", we set $r_{i}=\left\|M_{i}\right\|_{F}+0.15\left\|A_{i}^{T} A_{i}\right\|$ $(i=1,2,3)$, where $\|\cdot\|_{F}$ represents the Frobenius norm. For G-ADMM-S, we consider two cases of the matrices $G_{i}$ $(i=1,2,3)$ :

Case 1: $\quad G_{i}=r_{i} I_{n_{i} \times n_{i}}-\beta A_{i}^{T} A_{i} \quad$ with $\quad r_{i}=\left\|M_{i}\right\|_{F}+$ $0.15\left\|A_{i}^{T} A_{i}\right\|_{F}$

Case 2: $\quad G_{i}=r_{i} I_{n_{i} \times n_{i}}-\beta A_{i}^{T} A_{i} \quad$ with $\quad r_{i}=\left\|M_{i}\right\|_{F}+$ $\beta\left\|A_{i}^{T} A_{i}\right\|_{F}$

In order to investigate the stability and efficiency of our algorithms, we test 16 groups of problems with random data. Some preliminary numerical results are reported in Table 1 . Since they are synthetic examples with random date, for each scenario, we test 10 times and report the average performance. Specifically, we report the number of iterations ("Iter.") and the computing time in seconds ("Time") for all the tested methods. The data in Table 1 show that Algorithm G-ADMM-S
TABLE 1: Numerical results.

\begin{tabular}{lcccccc}
\hline & \multicolumn{2}{c}{ PPSM-C } & \multicolumn{3}{c}{ G-ADMM-S } \\
$\left(n_{1}, n_{2}, n_{3}\right)$ & \multicolumn{3}{c}{ Case 1} & \multicolumn{2}{c}{ Case 2} \\
& Iter. & Time & Iter. & Time & Iter. & Time \\
\hline$(500,500,500)$ & 1253 & 6.065 & 1589 & 6.290 & 1340 & 5.302 \\
$(500,600,500)$ & 1057 & 5.483 & 1744 & 7.628 & 1523 & 6.671 \\
$(600,500,600)$ & 1560 & 9.296 & 1825 & 8.589 & 1445 & 6.753 \\
$(600,600,600)$ & 1580 & 10.307 & 1915 & 10.142 & 1642 & 8.625 \\
$(600,700,600)$ & 1641 & 11.432 & 2042 & 11.817 & 1782 & 10.313 \\
$(700,600,700)$ & 1745 & 13.129 & 2223 & 13.157 & 1871 & 11.087 \\
$(700,700,700)$ & 2036 & 16.875 & 2357 & 16.304 & 1998 & 13.820 \\
$(700,800,700)$ & 2857 & 25.090 & 2628 & 19.335 & 2308 & 17.168 \\
$(800,700,800)$ & 2428 & 23.296 & 2634 & 19.875 & 2210 & 16.638 \\
$(800,800,800)$ & 2809 & 28.659 & 2804 & 23.453 & 2381 & 19.620 \\
$(800,900,800)$ & 4048 & 43.482 & 3016 & 28.678 & 2592 & 24.695 \\
$(900,800,900)$ & 3204 & 41.398 & 3178 & 31.030 & 2675 & 26.080 \\
$(900,900,900)$ & 3881 & 47.465 & 3332 & 36.036 & 2820 & 30.255 \\
$(900,1000,900)$ & 10000 & 129.147 & 3790 & 44.253 & 3224 & 37.557 \\
$(1000,900,1000)$ & 4547 & 71.910 & 3771 & 46.183 & 3140 & 38.250 \\
$(1000,1000,1000)$ & 10000 & 151.273 & 4131 & 53.813 & 3482 & 45.660 \\
\hline
\end{tabular}

$\left(G_{i}=r_{i} I_{n_{i} \times n_{i}}-\beta A_{i}^{T} A_{i}\right.$ with $\left.r_{i}=\left\|M_{i}\right\|_{F}+\beta\left\|A_{i}^{T} A_{i}\right\|_{F}\right)$ is more efficient than the rest of algorithms for the test problems.

5.2. Nonlinear Convex Programming Problem. In this section, we consider the following nonlinear convex programming problem:

$$
\begin{aligned}
& \min \left(\left\|x_{1}\right\|_{1}-\frac{1}{2} \ln \left(\left\|x_{1}\right\|^{2}+1\right)\right)+\left(q^{T} x_{2}+\frac{1}{2} x_{2}^{T} M x_{2}\right) \\
& \quad+\left(\frac{1}{2}\left\|x_{3}\right\|^{2}-\sum_{i=1}^{n_{3}} \cos x_{3, i}\right) \\
& \text { s.t. } \quad A_{1} x_{1}+A_{2} x_{2}+A_{3} x_{3}=b, \\
& x_{1} \in \mathscr{R}^{n_{1}}, 0 \leq x_{1}, x_{2} \in \mathscr{R}^{n_{2}}, x_{3} \in \mathscr{R}^{n_{3}},\left|x_{3, i}\right| \leq \frac{\pi}{2}, i=, 1, \ldots, n_{3},
\end{aligned}
$$

where $\|x\|_{1}=\sum_{i=1}^{n}\left|x_{i}\right|$ and $M$ is a positive matrix. In the experiments, we set $f_{1}\left(x_{1}\right)=\left\|x_{1}\right\|_{1}, g_{1}\left(x_{1}\right)=-\quad$ (1/ $2) \ln \left(\left\|x_{1}\right\|^{2}+1\right), f_{2}\left(x_{2}\right)=q^{T} x_{2}, g_{2}\left(x_{2}\right)=(1 / 2) x_{2}^{T} \quad M x_{2}, f_{3}$ $\left(x_{3}\right)=(1 / 2)\left\|x_{3}\right\|^{2}$, and $g_{3}\left(x_{3}\right)=-\sum_{i=1}^{n_{3}} \cos x_{3, i}$. It is easy to see that, when the PPSM-C in [25] solves (71), there is no explicit solution to the subproblems. In this section, we only use the Algorithm G-ADMM-S to solve (71). In the experiments, the entries of $A_{i} \in \mathscr{R}^{l \times n_{i}}(i=1,3)$ are uniformly distributed in $(0,1)$ with the density 0.1 and $l=n_{2}$. $x_{1}^{*}=0_{n_{1} \times 1}, x_{3}^{*}=0_{n_{3} \times 1}$, and $x_{2}^{*}$ is given generated by $x_{2}^{*}=$ sprand $\left(n_{2}, 1,0.5\right)$ in the Matlab function. We set the matrix $M$ in a way similar to $[25,31]$. In order to guarantee the feasibility of the problem, we set $q=-M x_{2}^{*}$ and $b=A_{1} x_{1}^{*}+A_{2} x_{2}^{*}+A_{3} x_{3}^{*}$. Thus, $\left(x_{1}^{*}, x_{2}^{*}, x_{3}^{*}\right)$ is an optimal solution of (71). The initial iteration points are the zero vectors $x_{i}^{0}=0_{n_{i} \times 1}(i=1,2,3)$ and $\lambda^{0}=0_{l \times 1}$ for all tested algorithms. We set a maximal number of 20000 for iteration of the proposed algorithms with a modified stopping criterion as follows: 
TABLe 2: Numerical results.

\begin{tabular}{|c|c|c|c|c|c|c|}
\hline \multirow{2}{*}{$\left(n_{1}, n_{2}, n_{3}\right)$} & \multicolumn{4}{|c|}{ Case $1(\mu=\beta)$} & \multicolumn{2}{|c|}{ Case $2(\mu=0.15)$} \\
\hline & Iter. & Time & f-error & Iter. & Time & f-error \\
\hline$(600,600,600)$ & 3760.0 & 42.487 & 0.000211 & 3535.6 & 39.895 & 0.000207 \\
\hline$(600,700,600)$ & 5036.0 & 68.516 & 0.000196 & 5205.0 & 71.399 & 0.000200 \\
\hline$(800,700,800)$ & 3598.0 & 59.179 & 0.000211 & 3842.8 & 64.059 & 0.000211 \\
\hline$(800,800,800)$ & 5620.0 & 109.576 & 0.000202 & 5114.4 & 99.910 & 0.000206 \\
\hline$(800,900,800)$ & 7206.0 & 170.436 & 0.000192 & 7015.2 & 158.227 & 0.000199 \\
\hline$(1000,900,1000)$ & 5655.0 & 148.923 & 0.000203 & 5351.4 & 140.452 & 0.000205 \\
\hline$(1000,1000,1000)$ & 7577.0 & 223.832 & 0.000202 & 7529.2 & 229.729 & 0.000198 \\
\hline
\end{tabular}

$\max \left\{\left\|x_{1}^{k}\right\|, \frac{\left\|x_{2}^{k}-\bar{x}_{2}^{k}\right\|}{\left\|x_{2}^{k}\right\|},\left\|x_{3}^{k}\right\|,\left\|A_{1} x_{1}^{k}+A_{2} x_{2}^{k}+A_{3} x_{3}^{k}-b\right\|\right\} \leq 10^{-3}$.

Now, we specify the choices of parameters to implement these algorithms. We set $H=\beta I$ with $\beta=0.01$, the relaxation parameter $\gamma=1.8, \quad r_{1}=n 1+\beta\left\|A_{1}^{T} A_{1}\right\|, \quad r_{2}=\|M\|_{F}+$ $\beta\left\|A_{2}^{T} A_{2}\right\|, \quad r_{3}=n 3+\beta\left\|A_{3}^{T} A_{3}\right\|, \quad$ and $G_{i}=r_{i} I_{n_{i} \times n_{i}}-\mu A_{i}^{T} A_{i}$ $(i=1,2,3)$. We consider two cases of the parameter $\mu$ : Case $1: \mu=\beta$; Case $2: \mu=0.15$.

We test 7 groups of problems with random data. Numerical results are reported in Table 2. For each scenario, we test 5 times and report the average performance. Specifically, we report the number of iterations ("Iter."), the computing time in seconds ("Time"), and the absolute error of function value ("f-error"). The numerical results show that Algorithm G-ADMM-S is effective.

\section{Conclusion}

In this paper, for the linearly constrained separable convex programming, whose objective function is the sum of $m$ individual blocks with nonoverlapping variables and each block is convex, we present a gradient-based ADMM with a substitution in the case $m \geq 3$. We have analysed its convergence and iteration complexity. The preliminary numerical results have shown the efficiency of the proposed algorithm.

\section{Data Availability}

No data were used to support this study.

\section{Conflicts of Interest}

The authors declare that they have no conflicts of interest.

\section{Acknowledgments}

The first author would like to extend his sincere gratitude to his doctoral supervisor, Pro. Caozong Cheng, for his guidance.

\section{References}

[1] D. Gabay and B. Mercier, "A dual algorithm for the solution of nonlinear variational problems via finite element approximation," Computers \& Mathematics with Applications, vol. 2, no. 1, pp. 17-40, 1976.

[2] R. Glowinski and A. Marroco, "Sur l'approximation, par éléments finis d'ordre un, et la résolution, par pénalisationdualité d'une classe de problèmes de Dirichlet non linéaires," Revue Française D'automatique, Informatique, Recherche Opérationnelle. Analyse Numérique, vol. 9, no. R2, pp. 41-76, 1975.

[3] S. Boyd, N. Parikh, E. Chu, B. Peleato, and J. Eckstein, "Distributed optimization and statistical learning via the alternating direction method of multipliers," Foundations and Trends in Machine Learning, vol. 3, no. 1, pp. 1-122, 2010.

[4] R. Glowinski, "On alternating direction methods of multipliers: a historical perspective," Computational Methods in Applied Sciences, vol. 34, pp. 59-82, 2014.

[5] M. Tao and X. M. Yuan, "Recovering low-rank and sparse components of matrices from incomplete and noisy observations," SIAM Journal on Optimization, vol. 21, no. 1, pp. 57-81, 2011.

[6] M. K. Ng, P. Weiss, and X. M. Yuan, "Solving constrained total-variation image restoration and reconstruction problems via alternating direction methods," SIAM Journal on Scientific Computing, vol. 32, no. 5, pp. 2710-2736, 2010.

[7] N. K. Bose and K. J. Boo, "High-resolution image reconstruction with multisensors," International Journal of Imaging Systems and Technology, vol. 9, no. 4, pp. 294-304, 1998.

[8] M. Ng, F. Wang, and X. M. Yuan, "Fast minimization methods for solving constrained total-variation superresolution image reconstruction," Multidimensional Systems and Signal Processing, vol. 22, no. 1-3, pp. 259-286, 2011.

[9] C. H. Rosa, Pathways of Economic Development in an Uncertain Environment: A Finite Scenario Approach to the U.S. Region under Carbon Emission Restrictions, WP-94-41, International Institute for Applied Systems Analysis, Laxenburg, Austria, 1994.

[10] S. Setzer, G. Steidl, and T. Teuber, "Deblurring Poissonian images by split Bregman techniques," Journal of Visual Communication and Image Representation, vol. 21, no. 3, pp. 193-199, 2010.

[11] V. Chandrasekaran, P. A. Parrilo, and A. S. Willsky, "Latent variable graphical model selection via convex optimization," The Annals of Statistics, vol. 40, no. 4, pp. 1935-1967, 2012.

[12] G. J. McLachlan, Discriminant Analysis and Statistical Pattern Recognition, p. 544, Wiley-Interscience, Hoboken, NJ, USA, 2004.

[13] L. F. Yang, J. Y. Luo, Y. Xu, Z. R. Zhang, and Z. Y. Dong, “A distributed dual consensus ADMM based on partition for DC-DOPF with carbon emission trading," IEEE Transactions on Industrial Informatics, vol. 16, no. 3, pp. 1858-1872, 2020.

[14] L. F. Yang, J. B. Jian, K. Meng, Y. Xu, and Z. Dong, "A novel projected two-binary-variable formulation for unit 
commitment in power systems," Applied Energy, vol. 187, pp. 732-745, 2017.

[15] M. T. Chao and C. Z. Cheng, "A note on the convergence of alternating proximal gradient method," Applied Mathematics and Computation, vol. 228, pp. 258-263, 2014.

[16] D. R. Han and X. M. Yuan, "A note on the alternating direction method of multipliers," Journal of Optimization Theory and Applications, vol. 155, no. 1, pp. 227-238, 2012.

[17] M. Y. Hong and Z. Q. Luo, "On the linear convergence of the alternating direction method of multipliers," Mathematical Programming, vol. 162, no. 1-2, pp. 165-199, 2017.

[18] C. H. Chen, B. S. He, Y. Ye, and X. M. Yuan, "The direct extension of ADMM for multi-block convex minimization problems is not necessarily convergent," Mathematical Programming, vol. 155, no. 1-2, pp. 57-79, 2016.

[19] M. Li, D. F. Sun, and K. C. Toh, "A convergent 3-block semiproximal ADMM for convex minimization problems with one strongly convex block," Asia-Pacific Journal of Operational Research, vol. 32, p. 19, 2015.

[20] D. F. Sun, K. C. Toh, and L. Q. Yang, “A convergent 3-block semiproximal alternating direction method of multipliers for conic programming with 4-type constraints," SIAM Journal on Optimization, vol. 25, no. 2, pp. 882-915, 2015.

[21] B. S. He, M. Tao, and X. M. Yuan, "Alternating direction method with Gaussian back substitution for separable convex programming," SIAM Journal on Optimization, vol. 22, no. 2, pp. 313-340, 2012.

[22] M. T. Chao, C. Z. Cheng, and D. Y. Liang, "A proximal block minimization method of multipliers with a substitution procedure," Optimization Methods and Software, vol. 30, no. 4, pp. 825-842, 2015.

[23] M. T. Chao, C. Z. Cheng, and H. B. Zhang, "A linearized alternating direction method of multipliers with substitution procedure, Asia Pac," Journal of Operations Research, vol. 32, p. 19, 2015.

[24] D. R. Han, X. M. Yuan, W. X. Zhang, and X. J. Cai, “An ADMbased splitting method for separable convex programming," Computational Optimization and Applications, vol. 54, no. 2, pp. 343-369, 2013.

[25] D. R. Han, H. J. He, and L. L. Xu, "A proximal parallel splitting method for minimizing sum of convex functions with linear constraints," Journal of Computational and Applied Mathematics, vol. 256, pp. 36-51, 2014.

[26] B. S. He, M. Tao, and X. M. Yuan, "A splitting method for separable convex programming," IMA Journal of Numerical Analysis, vol. 34, pp. 1-33, 2014.

[27] P. L. Lions and B. Mercier, "Splitting algorithms for the sum of two nonlinear operators," SIAM Journal on Numerical Analysis, vol. 16, no. 6, pp. 964-979, 1979.

[28] G. B. Passty, "Ergodic convergence to a zero of the sum of monotone operators in Hilbert space," Journal of Mathematical Analysis and Applications, vol. 72, no. 2, pp. 383-390, 1979.

[29] E. Blum and W. Oettli, Mathematische Optimierung, Econometrics and Operations Research XX, Springer-Verlag, New York, NY, USA, 1975.

[30] F. Facchinei and J. S. Pang, Finite-Dimensional Variational Inequalities and Complementarity Problems, Vol. I, Springer Series in Operations Research, Springer Verlag, New York, NY, USA, 2003.

[31] P. T. Harker and J. S. Pang, "A damped-Newton method for the linear complementarity problem," in Computational Solution of Nonlinear Systems of Equations, E. L. Allgower and
K. Georg, Eds., pp. 265-284, American Math. Soc., Providence, RI, USA, 1990. 